\title{
A switching observer for a class of non-uniformly observable systems via singular time-rescaling
}

\author{
Missie Aguado-Rojas, Trọng Biên Hoàng, William Pasillas-Lépine, Antonio Loría, and Witold Respondek
}

\begin{abstract}
We present a switching observer for a class of nonuniformly observable systems that are affine in the unmeasured states and nonlinear in the measured output. Using a singular time-rescaling, the dynamics of the estimation error is transformed into that of a bimodal switched linear system. Sufficient conditions that guarantee the observer's uniform asymptotic stability are provided; these are stated in terms of persistency of excitation and dwell-time of the (output-dependent) time-scaling function evaluated along the trajectories of the system. Unlike most results on switching observers, our approach does not rely on the solution of a set of linear matrix inequalities to compute the observer gain. In addition, we compare our scheme against the Kalman observer in a particular, but meaningful, case-study of observer-based control in automotive systems.
\end{abstract}

Index Terms-Observers, non-uniformly observable systems, switched systems, non-strict Lyapunov functions, dwell-time, persistency of excitation, time scaling, Kalman observer.

\section{INTRODUCTION}

Since the seminal work of Luenberger, for continuous timeinvariant linear systems, the observer design problem has been extensively studied in the literature and various approaches for different classes of nonlinear systems have been proposed see, e.g., [1]-[9] and references therein. In this note we address the observer design problem for non-uniformly observable systems, affine in the unmeasured states and nonlinear in the measured output. These are systems of the form

$$
\begin{aligned}
\dot{z} & =\gamma(y)[A z+d(y)]+b(y) u \\
y & =C z,
\end{aligned}
$$

where $z \in \mathbb{R}^{n}$ is the state, $y \in \mathbb{R}$ is the output, the pair $(A, C)$ is observable, and $\gamma: \mathbb{R} \rightarrow \mathbb{R}$ is a smooth function; we do not exclude output values $y \in \mathbb{R}$ such that $\gamma(y)=0$. Although systems (1) may appear restrictive at first sight, it is important to emphasize that many systems of the more general form

$$
\dot{x}=f(x)+g(x) u, \quad y=h(x),
$$

are equivalent to (1) up to a coordinate transformation, $z=$ $\Phi(x)$ where $x \in \mathbb{R}^{n}$. Conditions for the existence of such

This work was supported by the French National Research Agency (ANR) through the project HANDY, ANR-18-CE40-0010. The work of M. AguadoRojas was supported by the Mexican National Council of Science and Technology (CONACYT).

M. Aguado-Rojas is with L2S-CentraleSupélec, Université ParisSaclay, 91192 Gif-sur-Yvette, France. T. B. Hoàng is with Viettel Aerospace Institute, Hanoi, Vietnam. W. Pasillas-Lépine and A. Loría are with L2S-CentraleSupélec, CNRS, 91192 Gif-sur-Yvette, France (e-mail: william.pasillas-lepine@ centralesupelec.fr; antonio.loria@centralesupelec.fr). W. Respondek is with Laboratoire de Mathématiques de l'INSA Rouen, Normandie Université, 76800 Saint-Étienne-du-Rouvray, France (e-mail: witold.respondek@insa-rouen.fr). a transformation are given in [10], [11], and [12]. Beyond academic interest, however, our motivation to study systems of the form (1) comes from the fact that they appear in automotive control [13], mobile robotics [14], and chemical engineering [15], to mention a few applications — see also Section III.

Most observer-based schemes in the literature rely on the assumption that the system is uniformly observable. Yet, this is frequently untrue in many concrete control engineering problems [15], [16]. Indeed, in many cases $\gamma(y(t))$ may be equal (or tend) to zero, which leads to loss of observability. For instance, for initial states $z_{0} \in \mathbb{R}^{n}$ such that $\gamma\left(C z_{0}\right)=0$, the trajectories $z(t)$ generated via (1a) with $u \equiv 0$ satisfy $\gamma(y(t)) \equiv 0$ and are thus indistinguishable. For a more detailed description of the observability properties of this system, see [12, Prop. 1]. In the observer design for systems of the form (1), we allow for points such that $\gamma(y)=0$; this makes our main results more interesting for applications, but the respective proofs technically more involved. In our approach, the lack of uniform observability is overcome by constructing an $n$ dimensional switching observer, and imposing both persistency of excitation and zero-crossing dwell-time on the output.

Many other switching observers have been proposed in the literature, but mostly for switched linear systems [17]-[20]. They have also been used in several concrete applications, such as cellular processes [21], catalyst converters [22], mechanical systems [23], [24], and cyber-physical systems [25]. These approaches, however, do not provide a constructive procedure to design the observer gain. Instead, they rely on the existence of a solution for a set of linear matrix inequalities, from which the observer gain can be computed. Fundamentally different to designing observers for switched systems, in this note, switching is merely a technical mean to achieve the goal of designing an observer for non-uniformly observable systems.

Another technical building block is an output-dependent change of time-scale. In that regard, we borrow inspiration from the time-rescaling approach proposed in [10] and [11] - cf. [26], for the construction of observers for systems that cannot be linearized using a coordinate change only. As in the earliest work on time-rescaling [27], the time-scaling function considered in [10], [11], and [26] is regular and this restricts the construction of the proposed observers to uniformly observable systems. In this note, we use a singular time-scaling function (possibly not possessing a smooth inverse), thereby generalizing the observer design methodology of [11] to the realm of non-uniformly observable systems.

Now, it may be argued that constructing an observer for (1) is a simple task that has been thoroughly addressed in the literature. For instance, for the discretized version of this 
system, an observer can be designed using a Kalman filter $[28, \S 6.4]$. In a continuous-time setting, system (1) fits into the more general class of state-affine systems

$$
\dot{z}=A(t, u, y) z+B(t, u, y), \quad y=C z,
$$

studied, e.g., in [5], [29], [30]. Nevertheless, such approaches present several drawbacks. Using a Kalman-like construction as in [5], [29] involves solving a Riccatti ODE of dimension $n(n+1) / 2$ to determine the observer gain, which increases the complexity and the computation time necessary to implement the observer. Moreover, finding explicit conditions that guarantee the convergence of the observer is in general non trivial. In [30], for instance, weak sufficient conditions in terms of persistency of excitation of the output trajectories, $y(t)$, are given, but which are fairly difficult to verify.

To the best of our knowledge, we present the first switching observer that does not rely on solving a set of linear matrix inequalities to compute the observer gain. As a matter of fact, in contrast with other papers not relying on uniform observability, such as [30], we give an explicit expression for the observer gain that guarantees the asymptotic stability for the observer. Moreover, it is one of the few articles, along with [25], where a switching observer for a non-switched system is proposed. This note completes and builds upon the preliminary results presented in [12].

\section{OBSERVER DESIGN}

Since system (1) is affine in the unmeasured variables, a relatively simple manner to define a state observer is to mimic the Luenberger type, so let the state estimate $\hat{z}$ be defined by

$$
\dot{\hat{z}}=\gamma(y)[A \hat{z}+d(y)+K(y)(y-C \hat{z})]+b(y) u,
$$

where the column vector $K(\cdot)$ depends on the system's output. The difficulty of such design resides in defining the observer gain $K(y)$ such that the estimation error, $e:=\hat{z}-z$, tends to zero in spite of the loss of observability at the instants $t$ such that $\gamma(y(t))=0$.

Furthermore, beyond mere convergence of the estimation error, the problem that we address is that of designing $K(\cdot)$ such that the origin, for the estimation error dynamics,

$$
\dot{e}=\gamma(y)[A-K(y) C] e,
$$

is globally asymptotically stable, uniformly in $y-c f$. [30].

To that end, we proceed according to the following reasoning. Let us assume temporarily that the function $\gamma$ is sign-definite, that is, either $\gamma(y)>0$ or $\gamma(y)<0$ for all $y \in \mathbb{R}$. Akin to [11], in such case, we may define a new time variable $\tau$, such that

$$
\frac{d \tau}{d t}=\left\{\begin{aligned}
\gamma(y(t)) & \text { if } \gamma(y(t))>0 \\
-\gamma(y(t)) & \text { if } \gamma(y(t))<0
\end{aligned}\right.
$$

Then, in the new time scale defined by $\tau$, the estimation error dynamics becomes

$$
\frac{d e}{d \tau}=\left\{\begin{aligned}
{[A-K(y) C] e } & \text { if } \gamma(y)>0 \\
-[A-K(y) C] e & \text { if } \gamma(y)<0
\end{aligned}\right.
$$

so, to ensure the stability of the origin for (5), depending on whether $\gamma(y)>0$ or $\gamma(y)<0$, the gain $K(y)$ can be defined as a constant matrix $K$ such that $A-K C$ or $-[A-K C]$ is Hurwitz.

Assuming that $\gamma(y)$ is sign-definite rules out the existence of the singular set $\{\gamma(y)=0\}$, where the system looses its observability [12, Prop. 1]. Nevertheless, this assumption is clearly over-conservative and precludes the use of the proposed observer (2) for some concrete examples of control systems of the form (1) $-c f$. [13]. Therefore, as in [12], in this note we consider the scenario in which $\gamma(y(t))$ may vanish or change sign at some time-instances $t$. Note that such scenario imposes the technical difficulty of overcoming the lack of observability for values of $y(t) \in \mathbb{R}$ such that $\gamma(y(t))=0$. Roughly speaking, our main result establishes that the signindefiniteness of $\gamma(y)$ may be overcome by using a switchingbased observer design that we describe next.

Choose two constant vectors:

$$
K_{+}=\left[\begin{array}{lll}
k_{1}^{+} & \ldots & k_{n}^{+}
\end{array}\right]^{\top}, \quad K_{-}=\left[\begin{array}{lll}
k_{1}^{-} & \ldots & k_{n}^{-}
\end{array}\right]^{\top}
$$

such that the matrices

$$
\begin{aligned}
& A_{+}:=A-K_{+} C, \\
& A_{-}:=-A+K_{-} C
\end{aligned}
$$

are Hurwitz and let us define

$$
K(y(t))= \begin{cases}K_{+}, & \text {if } \gamma(y(t))>0 \\ K_{-}, & \text {if } \gamma(y(t))<0\end{cases}
$$

Then, the dynamics of the estimation error, $e$, in the $\tau$ timescale, becomes

$$
\frac{d e}{d \tau}=\left\{\begin{aligned}
{\left[A-K_{+} C\right] e, } & \text { if } \gamma(y(t))>0 \\
-\left[A-K_{-} C\right] e, & \text { if } \gamma(y(t))<0
\end{aligned}\right.
$$

which is a switched system. Indeed, in compact form, we have

$$
\frac{d e}{d \tau}=\mathcal{A}_{\sigma(\tau)} e,
$$

where $\sigma: \mathbb{R}_{\geq 0} \rightarrow\{+1,-1\}$ is a switching function defined farther below (see Lemma 2 ), $\mathcal{A}_{+1}:=A_{+}$, and $\mathcal{A}_{-1}:=A_{-}$.

Equation (9) represents a switched system that may be analyzed using, for example, the methods proposed in [31]. To that end, a potential approach is to choose two constant vectors $K_{+}$and $K_{-}$such that $A_{+}$and $A_{-}$in (6) and (7), respectively, are Hurwitz and, in addition, to choose those vectors in such a way that the switched system (9) admits a common (non-strict) Lyapunov function (valid for both $A_{+}$and $A_{-}$), in order to ensure exponential stability of the estimation error dynamics, albeit, in the $\tau$ time-scale. This brings us to our first statement.

Theorem 1: Let $(A, C)$ be an observable pair, define $Q=$ $C^{\top} C$ and consider the matrices defined in (6), (7). If $K_{+}$is such that $A_{+}$is Hurwitz, then there exists a unique gain $K_{-}$ such that $A_{-}$is also Hurwitz and the Lyapunov equations

$$
\begin{aligned}
& A_{+}^{\top} P+P A_{+}=-Q \\
& A_{-}^{\top} P+P A_{-}=-Q
\end{aligned}
$$

admit a common solution $P=P^{\top}>0$.

The following remarks are in order. Theorem 1 is essentially contained in the preliminary work [12] —in the latter it is not stated that the matrix $A_{-}$is also Hurwitz, but an explicit 
solution for $P$ is given and it is also shown that if the pair $(A, C)$ is in the observable canonical form, then the elements of $K_{-}$are given by

$$
k_{i}^{-}:=(-1)^{i} k_{i}^{+}+\left[1-(-1)^{i}\right] a_{i}, \quad \forall 1 \leq i \leq n .
$$

We also remark that even though both $A_{+}$and $A_{-}$are Hurwitz, the matrix $Q$ in (10)-(11) is only positive semidefinite. As a matter of fact, it is known from [32] that these Lyapunov equations do not admit a common solution $P$ if $n=2$ and $Q$ is positive definite. Therefore, Theorem 1 is a statement of interest in its own right. The methods proposed in [31] provide a straightforward stability analysis for (9), assuming that one disposes of a (non-strict) Lyapunov function. Nevertheless, for an arbitrary switched system, we are not aware of any statement that asserts the existence of a common Lyapunov function, let alone a general analytical method to construct it. In the particular case of (9), Theorem 1 not only guarantees the existence of this common function for particular values of the gains $K_{+}$and $K_{-}$, but its proof — see [12]— provides an analytic expression of $P$.

Now, although intuitive, the rationale that leads to equation (9) hides several technical difficulties. Firstly, it relies on the ability of defining the new time variable $\tau$ which, in order to make sense as such, must be well-defined. That is, as a function of $t$, the new time $\tau$ must be continuous, strictly increasing, and radially unbounded. Secondly, strictly speaking, system (3) is non-autonomous as it depends on the output trajectories $y\left(t, t_{0}, z_{0}\right)$. Thus, rather than defining $\tau$ as in (4) we define it, more precisely for each $\left(t_{0}, z_{0}\right)$, as

$$
\tau=\nu\left(t, t_{0}, z_{0}\right)=\int_{t_{0}}^{t}\left|\gamma\left(y\left(s, t_{0}, z_{0}\right)\right)\right| d s,
$$

which satisfies

$$
\frac{d \tau}{d t}=\left|\gamma\left(y\left(s, t_{0}, z_{0}\right)\right)\right|
$$

This means that $\tau$ is not only a function of $t$, but it is inherently parameterized by the system's initial conditions $\left(t_{0}, z_{0}\right)$. Therefore, appropriate technical conditions, stated below as Assumption 1, must be imposed in order to establish the uniform global asymptotic stability of the origin for (3).

In writing (13), it is implicitly assumed that the trajectories are forward complete. As a matter of fact, following standard practice in the literature on observer design, it is assumed that each pair of initial conditions $\left(t_{0}, z_{0}\right) \in \mathbb{R} \times \mathbb{R}^{n}$ and each input $u(\cdot)$ generates a unique (smooth) trajectory $z\left(t, t_{0}, z_{0}, u(\cdot)\right)$ that is uniformly bounded in $t_{0} \in \mathbb{R}$. To avoid a cumbersome notation, however, in the sequel we drop the argument $u$ by considering trajectories generated by a fixed, but arbitrary, input.

Assumption 1: The function $\gamma$ in (1a) and the output trajectories, $y\left(t, t_{0}, z_{0}\right)$, satisfy the following conditions:

(persistency of excitation) there exist $\mu_{0}>0$ and $T_{0}>0$ such that, for all $\left(t_{0}, z_{0}\right) \in \mathbb{R} \times \mathbb{R}^{n}$,

$$
\int_{t}^{t+T_{0}} \gamma\left(y\left(s, t_{0}, z_{0}\right)\right)^{2} d s \geq \mu_{0}, \quad \forall t \geq t_{0} ;
$$

(dwell-time) there exists $T_{D}>0$ such that, for any $\left(t_{0}, z_{0}\right) \in \mathbb{R} \times \mathbb{R}^{n}$ and any two instants $t_{k} \neq t_{l}$ satisfying $\gamma\left(y\left(t_{k}, t_{0}, z_{0}\right)\right)=\gamma\left(y\left(t_{l}, t_{0}, z_{0}\right)\right)=0$,

$$
\left|t_{k}-t_{l}\right| \geq T_{D}
$$

Remark 1: Both conditions, (14) and (15), are required to hold uniformly in the output trajectories, hence, in $t_{0}$ and compact sets of initial conditions $z_{0}$. The persistency of excitation condition cannot, in general, be verified analytically, but it is common in the literature (even in scenarii involving output feedback control) because of its clear meaning and of its importance as a necessary condition for uniform global asymptotic stability. It holds, for instance, if $t \mapsto \gamma(y(t))$ is oscillatory. Also, we stress the second part of Assumption 1 does not define dwell-time -for this see, e.g., [31]—, but $T_{D}$ gives a lower bound on the time between two consecutive switches of the sign of $\gamma(y(t))$.

Assumption 1 ensures that the change of time (13) is defined via a globally invertible function. More precisely, the following statement holds - the proof is presented in Section IV.

Lemma 1: Consider a continuous function $y \mapsto \gamma(y)$ and an absolutely continuous function $t \mapsto y\left(t, t_{0}, z_{0}\right)$, bounded uniformly in $t_{0}$ and satisfying Assumption 1. Then, for each pair $\left(t_{0}, z_{0}\right)$, the function $\nu\left(\cdot, t_{0}, z_{0}\right):\left[t_{0},+\infty\right) \mapsto[0,+\infty)$, defined by (13), is continuous, strictly increasing, and radially unbounded. It is thus globally invertible. Moreover, for each $\left(t_{0}, z_{0}\right), \nu\left(t, t_{0}, z_{0}\right) \rightarrow \infty$ as $t \rightarrow \infty$, uniformly in $t_{0}$. $\triangleleft$

Furthermore, the dwell-time condition (15) ensures that the time instants at which the observability singularity $\{\gamma(y)=0\}$ is crossed do not accumulate. This condition is fundamental for the following reasons. For the purpose of implementation, according to (8), the value of the observer gain is assigned by verifying whether the sign of $\rho(t):=\gamma(y(t))$ is positive or negative. In turn, this defines a switching signal in the natural time-scale, i.e., with respect to $t$. For the purpose of analysis, however, the system's behavior is considered as defined in the transformed time-scale, with respect to $\tau$. The following statement, whose proof is also provided in Section IV, ensures that the dwell-time condition is preserved under the time-scale transformation.

Lemma 2: Let $\gamma(y)$ and $y\left(t, t_{0}, z_{0}\right)$ satisfy Assumption 1 and consider the function $\nu\left(\cdot, t_{0}, z_{0}\right)$ defined in (13). Let $\rho$ : $\mathbb{R} \rightarrow\{-1,+1\}$ and $\sigma: \mathbb{R}_{\geq 0} \rightarrow\{-1,+1\}$ be two functions related by $\sigma(\tau)=\rho \circ \nu^{-1}(\tau)$. If $t \mapsto \rho(t)$ has a dwell-time, then so does $\tau \mapsto \sigma(\tau)$.

Furthermore, by imposing $T_{D}$ to be independent of the output trajectory that generates each switching sequence, it is ensured that stability and convergence for the switched system in the $\tau$ time-scale remain uniform in the natural time-scale. Our main statement, presented below, relies on Theorem 1 and Lemmata 1 and 2; its proof is presented in Section IV.

Theorem 2 (main result): Consider system (1), with $(A, C)$ observable and under Assumption 1. Consider also the observer given by (2) and (8), with $K_{+}$such that $A-K_{+} C$ is Hurwitz. Then, there exists $K_{-}$such that, for the estimation error dynamics (3), the origin is globally asymptotically stable, uniformly in the output trajectories. Furthermore, for 
a pair $(A, C)$ in observable companion form, the elements of $K_{-}:=\left[\begin{array}{lll}k_{1}^{-} & \cdots & k_{n}^{-}\end{array}\right]$may be taken according to (12).

\section{Application EXAMPLE}

In this section, we illustrate the performance of the proposed observer on a concrete example of automotive control; specifically, concerning the antilock braking system (ABS). The objective is to estimate the unmeasurable tyre extended braking stiffness (XBS) - a variable closely related to the adhesion coefficient between the wheel and the road - through measurements of the acceleration of the wheel and the vehicle. The interest in doing so is that using the XBS one may control the ABS so as to maximize tyre-road adherence. For an indepth discussion on this problem, see e.g., [13] and [16].

The dynamics of the system is described by

$$
\begin{aligned}
{\left[\begin{array}{c}
\dot{z}_{1} \\
\dot{z}_{2}
\end{array}\right] } & =\frac{z_{1}}{v_{x}(t)}\left(\left[\begin{array}{cc}
0 & \theta_{1} \\
0 & \theta_{3}
\end{array}\right]\left[\begin{array}{c}
z_{1} \\
z_{2}
\end{array}\right]+\left[\begin{array}{c}
0 \\
\theta_{4}
\end{array}\right]\right)+\left[\begin{array}{c}
\theta_{2} \\
0
\end{array}\right] u, \\
y & =z_{1}
\end{aligned}
$$

where the measured output $z_{1}$ is the difference between the linear acceleration of the wheel and the longitudinal acceleration of the vehicle, $z_{2}$ is the XBS, $u$ is the time derivative of the brake pressure, $\theta_{1}$ and $\theta_{2}$ are parameters that depend on the wheel and the brake actuator, $\theta_{3}$ and $\theta_{4}$ are parameters that depend on the road conditions (all the parameters $\theta_{i}$ are assumed to be known and constant), and $v_{x}(t)$ is the longitudinal speed of the vehicle. The latter is assumed to be strictly positive and separated from zero and it is considered as a known external variable. This is important because, then, the system (16) may be considered as in the form (1) with $\gamma(y):=y$. To better see this, replace the original time $d t$ with $d t / v_{x}(t)$ and the control input $u$ with $u v_{x}(t)$.

The performance of the switching observer is illustrated in Figure 1. The simulation scenario corresponds to an ABS braking maneuver of a vehicle traveling on dry asphalt with an initial speed of $120 \mathrm{~km} / \mathrm{h}$. The system's parameters are $\theta_{1}=562.5 \mathrm{~N} \cdot \mathrm{kg}^{-1}, \theta_{2}=4.37 \mathrm{~N} \cdot \mathrm{kg}^{-1} \cdot \mathrm{bar}^{-1}, \theta_{3}=23.99$, and $\theta_{4}=12.47$. The control input is defined by a hybrid ABS controller [33] that generates an oscillatory output trajectory (see Figure 1a), hence Assumption 1 holds. The estimation results of the switching observer are compared with respect to those obtained via a Kalman observer (see [5, Th. 4]).

Both observers provide a good estimation of the acceleration offset, despite the measurement of the latter being perturbed by a zero-mean white noise with a standard deviation $\sigma=4.5 \mathrm{~m} / \mathrm{s}^{2}$, typical in an ABS [13]. Concerning the estimation of the XBS, however, the switching observer clearly outperforms the Kalman observer. With the switching observer the estimation of $z_{2}$ exhibits a slight deviation from its true value whenever the latter goes from the troughs of the waveform towards zero, whereas with the Kalman observer the estimation exhibits a much larger deviation (starting at the crest of the waveform) and for a longer amount of time. Moreover, we remark that a correct estimation of the time instants in which $z_{2}$ crosses zero is of critical importance for the control of the ABS, thus rendering the Kalman observer unsuited for this particular application.
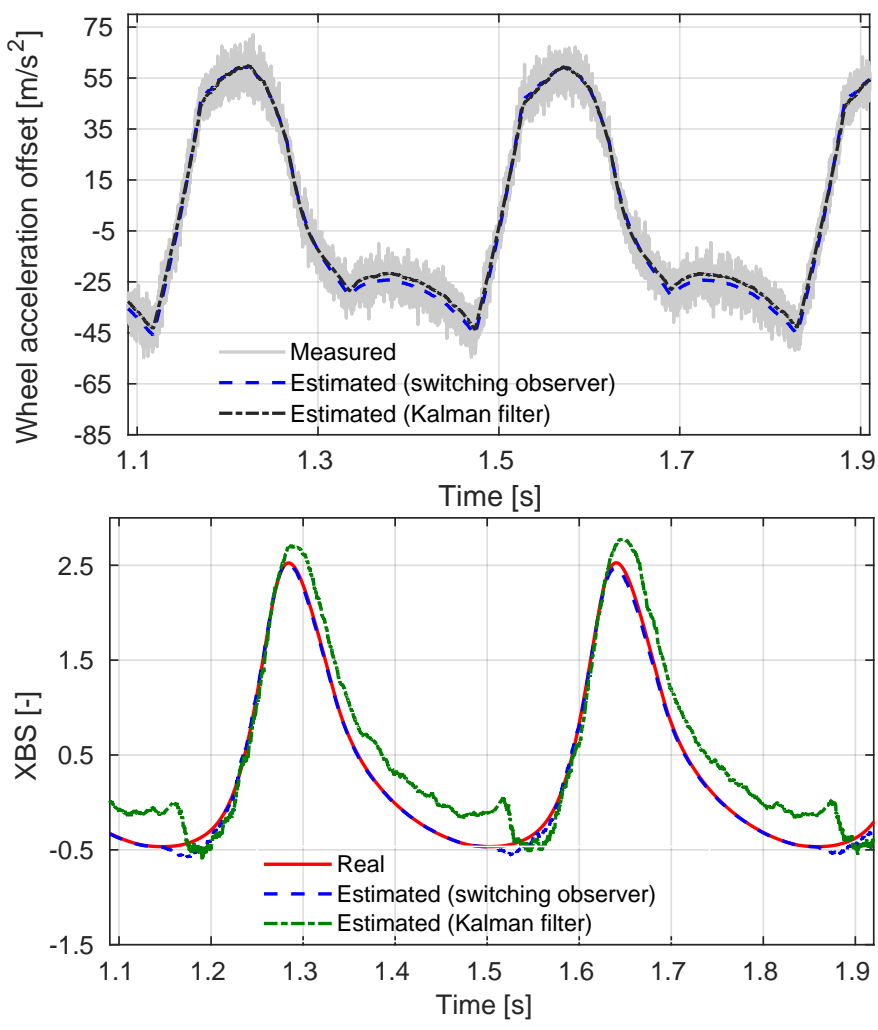

Fig. 1. Comparison between the proposed switching observer and the well-known Kalman observer: application to the antilock braking system. TOP: Measurement and estimation of the acceleration offset, $z_{1}$. ВотTOM: Estimation of the tyre extended braking stiffness, $z_{2}$.

\section{Proofs}

\section{A. Proof of Theorem 1}

Existence: Given $K_{+}$such that $A_{+}=A-K_{+} C$ is Hurwitz and $Q=C^{\top} C$, let $P$ denote the unique solution of (10). Then, $K_{-}=K_{+}-P^{-1} C^{\top}$ is such that $P$ satisfies (11). The matrix $A_{-}:=-A+K_{+} C$ is necessarily Hurwitz, because $\left(A_{-}, C\right)$ is observable (see, e.g., [34, Prop. 5.4]).

Uniqueness: Assume, without loss of generality, that the pair $(A, C)$ is in observable companion form. Now, to examine whether there exist other gains $K_{-}$such that $P$ satisfies (11), we start by observing that such $P$ necessarily satisfies

$$
A_{0}^{\top} P+P A_{0}=-Q, \quad \text { with } \quad A_{0}=\frac{1}{2}\left(A_{+}+A_{-}\right) \text {. }
$$

This is because $A_{0}$ belongs to the matrix pencil generated by $A_{+}$and $A_{-}$. Premultiplying both sides of the first equation in (17) by $P^{-1}$, we obtain

$$
P^{-1} A_{0}^{\top} P+A_{0}=-P^{-1} Q .
$$

Then, observing that $A_{0}^{\top} P=P A_{0}$ and premultiplying both sides of this expression by $P^{-1}$, we obtain

$$
P^{-1} A_{0}^{\top} P=A_{0} .
$$

From (18) and (19) we see that $A_{+}+A_{-}=-P^{-1} Q$, i.e.,

$$
\left(K_{+}-K_{-}\right) C=-P^{-1} Q,
$$

whose unique solution is $K_{-}=K_{+}-P^{-1} C^{\top}$. 


\section{B. Proof of Theorem 2}

Let $z_{0} \in \mathbb{R}^{n}$ and $t_{0} \in \mathbb{R}$ be arbitrarily fixed initial conditions generating, through (1a), the state trajectories $z\left(t, t_{0}, z_{0}\right)$ and, through $(1 \mathrm{~b})$, the output trajectory $y\left(t, t_{0}, z_{0}\right)=C z\left(t, t_{0}, z_{0}\right)$. Then, consider the estimation error equation

$$
\dot{e}=\gamma\left(y\left(t, t_{0}, z_{0}\right)\right)\left[A-K\left(\gamma\left(y\left(t, t_{0}, z_{0}\right)\right)\right) C\right] e
$$

with initial conditions $\left(t_{0}, e_{0}\right)$, where $e_{0}=\hat{z}_{0}-z_{0}$.

In view of the dwell-time condition in Assumption 1, the time instants $\left\{t_{k}\right\}$ where $\gamma\left(y\left(t_{k}, t_{0}, z_{0}\right)\right)=0$ do not accumulate, regardless of $\left(t_{0}, z_{0}\right)$. Therefore, and since $t_{0}$ and $z_{0}$ are arbitrarily fixed, for all $t \neq t_{k}$, we may define, with a mild abuse of notation, $\rho(t):=\operatorname{sgn}\left(\gamma\left(y\left(t, t_{0}, z_{0}\right)\right)\right)$, where $\operatorname{sgn}(\cdot)$ takes values in $\{-1,+1\}$. It follows that, for almost all $t \geq t_{0}$,

$$
\frac{\dot{e}}{|\gamma(y(t))|}=\rho(t)[A-K(\gamma(y(t))) C] e,
$$

where, for simplicity, we write $y(t)$ instead of $y\left(t, t_{0}, z_{0}\right)$. The solutions of (21) coincide with those of (20) for all $t \geq t_{0}$ and are absolutely continuous. Furthermore, let $\tau=\nu\left(t, t_{0}, z_{0}\right)$ be defined as in (13), then $\tau_{0}:=\nu\left(t_{0}, t_{0}, z_{0}\right)$ satisfies $\tau_{0}=$ 0 . Moreover, note that in view of Lemma $1, \nu\left(\cdot, t_{0}, z_{0}\right)$ is continuous, strictly increasing, and radially unbounded. This, in turn, implies that $t=\nu^{-1}\left(\cdot, t_{0}, z_{0}\right)$ exists and is continuous on $[0, \infty)$. Let $\bar{y}\left(\tau, z_{0}\right) \equiv y\left(t, t_{0}, z_{0}\right)$, that is, we denote by $\bar{y}\left(\tau, z_{0}\right)$ the system's output trajectories in the $\tau$ time-scale.

It follows that for each $e_{0}$ the system

$$
\frac{d e}{d \tau}=\sigma(\tau)\left[A-K\left(\gamma\left(\bar{y}\left(\tau, z_{0}\right)\right)\right) C\right] e,
$$

where $\sigma$ is defined in Lemma 2, generates solutions $e\left(\cdot, e_{0}\right)$ that are absolutely continuous functions of $\tau$.

Now, by assumption, $A_{+}$in (6) is Hurwitz, so by Theorem 1 we obtain the unique $K_{-}$such that $A_{-}$is also Hurwitz and, moreover, such that the respective Lyapunov equations (10), (11) admit a common solution $P=P^{\top}>0$. Thus, define

$$
\mathcal{A}_{\sigma(\tau)}= \begin{cases}A_{+} & \text {if } \sigma(\tau)>0 \\ A_{-} & \text {if } \sigma(\tau)<0\end{cases}
$$

It follows, from Assumption 1 and Lemma 1, that the solutions of the estimation error equation (20) coincide with those of the linear switched system described by (9) and (22), with $\tau_{0}=0$.

Furthermore, in view of the dwell-time condition (15) and by virtue of Lemma 2, the switching signal $\sigma$ has a dwell time. Therefore, from [31, Th. 4], it follows that the origin for the system described by (9) and (22) is globally exponentially stable, uniformly with respect to the switching signals. In view of the continuity and invertibility of $\tau$ (see the proof of Lemma 2 below) it follows that $e=0$ is globally asymptotically stable. Uniformity follows from the fact that the previous developments hold regardless of $t_{0}$.

The second statement is a direct consequence of Theorem 1 and so this concludes the proof of Theorem 2 .

\section{Proofs of Lemmata 1 and 2}

Proof of Lemma 1: Let $\left(t_{0}, z_{0}\right)$ be arbitrarily fixed. Then, with a slight abuse of notation, in the sequel we drop these arguments when clear from the context. From (13) we see that $\nu(t)>0$ for all $t \geq t_{0}$ and $\nu\left(t_{0}\right)=0$. Continuity follows directly from that of $\gamma$ and absolute continuity of $y\left(\cdot, t_{0}, z_{0}\right)$. To show that $\nu$ is strictly increasing we proceed by contradiction. If $\nu$ is not strictly increasing, there exist an interval $[a, b] \subset\left[t_{0}, \infty\right)$ and a constant $c>0$ such that $\nu(t)=c$ for all $t \in[a, b]$ or, equivalently, $\dot{\nu}(t)=|\gamma(y(t))|=0$ on the same interval. Nonetheless, this contradicts the dwell-time condition in Assumption 1 for any $T_{D} \leq b-a$. Moreover, this holds regardless of $\left(t_{0}, z_{0}\right) \in \mathbb{R} \times \mathbb{R}^{n}$.

Now, because $y$ is uniformly bounded and $\gamma$ is continuous, $\gamma\left(y\left(\cdot, t_{0}, z_{0}\right)\right)$ is also bounded, uniformly for all $t_{0} \in \mathbb{R}$ and all $\left|z_{0}\right|<r$, and for any $r>0$. That is, for any $r>0$ there exists $\gamma_{\max }(r)>0$ such that $\left|\gamma\left(y\left(t, t_{0}, z_{0}\right)\right)\right| \leq \gamma_{\max }(r)$ for all $t \geq t_{0}$ and all $\left|z_{0}\right|<r$. This implies that

$$
\int_{t_{0}}^{t}\left|\gamma\left(y\left(s, t_{0}, z_{0}\right)\right)\right| d s \leq \gamma_{\max }(r)\left[t-t_{0}\right]
$$

and, from (13), it follows that $\nu(t) \leq \gamma_{\max }(r)\left[t-t_{0}\right]$. Hence, $\nu$ is defined on $\left[t_{0}, \infty\right)$. Furthermore, because $|\gamma(y(t))| \leq$ $\gamma_{\max }(r)$, we also have

$$
\left|\frac{\gamma(y(t))}{\gamma_{\max }(r)}\right| \geq\left[\frac{\gamma(y(t))}{\gamma_{\max }(r)}\right]^{2}
$$

so, multiplying both sides of the expression above by $\left(\gamma_{\max }(r)\right)^{2}$ and integrating from $t$ to $t+T_{0}$, we obtain

$\int_{t}^{t+T_{0}} \gamma_{\max }(r)\left|\gamma\left(y\left(s, t_{0}, z_{0}\right)\right)\right| d s \geq \int_{t}^{t+T_{0}} \gamma\left(y\left(t, t_{0}, z_{0}\right)\right)^{2} d s$.

In turn, from the latter and the persistency-of-excitation condition (14), it follows that, for any $n \in \mathbb{N}$,

$$
\int_{t}^{t+n T_{0}}\left|\gamma\left(y\left(s, t_{0}, z_{0}\right)\right)\right| d s \geq n \frac{\mu_{0}}{\gamma_{\max }(r)} .
$$

Evaluating the limit as $n \rightarrow+\infty$ on both sides of the latter inequality and comparing it to (13), we see that

$$
\lim _{t \rightarrow \infty} \nu(t) \geq \lim _{n \rightarrow \infty} \int_{t_{0}}^{t_{0}+n T_{0}}\left|\gamma\left(y\left(s, t_{0}, z_{0}\right)\right)\right| d s=+\infty
$$

The fact that $\mu_{0}$ and $T_{0}$ are independent of $t_{0}$ guarantees that the limit above holds uniformly in $t_{0}$.

Proof of Lemma 2: We proceed by contradiction. Assume that $t \mapsto \rho(t)$ has a dwell time, but $\tau \mapsto \sigma(\tau)$ does not. Therefore, for any $\varepsilon>0$ there exists $\tau_{k}$ and $\tau_{l}$ such that $\tau_{k} \neq \tau_{l}$ and $\gamma\left(y\left(\tau_{k}\right)\right)=\gamma\left(y\left(\tau_{l}\right)\right)=0$ and $\left|\tau_{k}-\tau_{l}\right|<\varepsilon$. Next, let $\left\{\varepsilon_{n}\right\}$ be a sequence converging to zero and, for each $\varepsilon_{n}$, consider a pair $\left(\tau_{k}^{n}, \tau_{l}^{n}\right)$ as defined previously. That is, let the converging sequence $\left\{\varepsilon_{n}\right\}$ generate a sequence $\left\{\left(\tau_{k}^{n}, \tau_{l}^{n}\right)\right\}$. Now, in view of Lemma $1, t \mapsto \nu(t)$ is continuous, strictly increasing and radially unbounded. Therefore, $\tau \mapsto \nu^{-1}(\tau)$ exists and is continuous on $[0,+\infty)$. Then, set $\delta_{n}:=\left|\nu^{-1}\left(\tau_{k}^{n}\right)-\nu^{-1}\left(\tau_{l}^{n}\right)\right|$. It follows, from continuity of $\nu^{-1}$, that the sequence $\left\{\varepsilon_{n}\right\}$ generates another converging sequence $\left\{\delta_{n}\right\}$, which may be equivalently written as $\left\{\left|t_{k}^{n}-t_{l}^{n}\right|\right\} \rightarrow 0$. The latter, however, contradicts the premise that $t \mapsto \rho(t)$ has a dwell time. 


\section{Conclusions}

The design of our switching observer for non-uniformly observable systems relies on a singular time-rescaling approach to transform the estimation error dynamics into a linear system whose dynamics switches between two stable modes. Although it was illustrated, through a concrete practical example, that the approach may supersede Kalman-based designs, several interesting theoretical questions remain open. One pertains to the optimal choice of the observer gains for each stable mode. For instance, one may consider setting $K_{+}$to the value given by the minimum mean-square linear estimator, which is computed using the solution of an algebraic Riccati equation. Nevertheless, the conditions that guarantee both the solvability of the Riccati equation and equations (10) and (11) are not straightforward. Identifying the systems for which this optimal choice generates a common Lyapunov function is an interesting question to study. Furthermore, it seems interesting to explore the links between Theorem 1 and the back-andforth-nudging technique [35], in which one is confronted to a similar problem of guaranteeing the stability of a system that is iteratively integrated forward and backward in time.

\section{ACKNOWLEDGEMENTS}

The authors are grateful to an anonymous reviewer who pointed out the short proof of existence of $K_{-}$in Theorem 1 .

\section{REFERENCES}

[1] A. J. Krener and A. Isidori, "Linearization by output injection and nonlinear observers," Systems and Control Letters, vol. 3, no. 1, pp. 47-52, 1983.

[2] A. J. Krener and W. Respondek, "Nonlinear observers with linearizable error dynamics," SIAM Journal on Control and Optimization, vol. 23, no. 2, pp. 197-216, 1985.

[3] J.-P. Gauthier and I. Kupka, Deterministic Observation Theory and Applications. Cambridge University Press, 2001.

[4] A. Radke and Z. Gao, "A survey of state and disturbance observers for practitioners," in 2006 American Control Conference, 2006, pp. 51835188.

[5] G. Besançon, "An overview on observer tools for nonlinear systems," in Nonlinear observers and applications, ser. Lecture Notes in Control and Information Sciences, G. Besançon, Ed. Springer-Verlag: Berlin Heidelberg, 2007, vol. 363, pp. 1-33.

[6] P. Dufour, S. Flila, and H. Hammouri, "Observer design for MIMO non-uniformly observable systems," IEEE Transactions on Automatic Control, vol. 57, no. 2, pp. 511-516, 2012.

[7] M. Farza, T. Ménard, A. Ltaief, I. Bouraoui, M. MSaad, and T. Maatoug, "Extended high gain observer design for a class of MIMO non-uniformly observable systems," Automatica, vol. 86, pp. 138-146, 2017.

[8] P. Bernard, L. Praly, V. Andrieu, and H. Hammouri, "On the triangular canonical form for uniformly observable controlled systems," Automatica, vol. 85, pp. 293-300, 2017.

[9] L. Menini, C. Possieri, and A. Tornambe, "Observers for linear systems by the time-integrals and moving average of the output," IEEE Transactions on Automatic Control, 2019.

[10] M. Guay, "Observer linearization by output-dependent time-scale transformations," IEEE Transactions on Automatic Control, vol. 47, no. 10, pp. 1730-1735, 2002.

[11] W. Respondek, A. Pogromsky, and H. Nijmeijer, "Time scaling for observer design with linearizable error dynamics," Automatica, vol. 40, no. 2, pp. 277-285, 2004

[12] T. B. Hoàng, W. Pasillas-Lépine, and W. Respondek, "A switching observer for systems with linearizable error dynamics via singular time-scaling," in Proceedings of the 21st International Symposium on Mathematical Theory of Networks and Systems, 2014, pp. 1101-1108.
[13] T. B. Hoàng, W. Pasillas-Lépine, A. De Bernardinis, and M. Netto, "Extended braking stiffness estimation based on a switched observer, with an application to wheel-acceleration control," IEEE Transactions on Control Systems Technology, vol. 22, no. 6, pp. 2384-2392, 2014.

[14] E. Lefeber, A. Robertsson, and H. Nijmeijer, "Linear controllers for exponential tracking of systems in chained-form," International Journal of Robust and Nonlinear Control, vol. 10, no. 4, pp. 243-263, 2000.

[15] A. Rapaport and D. Dochain, "A robust asymptotic observer for systems that converge to unobservable states - a batch reactor case study," IEEE Transactions on Automatic Control, vol. 65, no. 6, pp. 2693-2699, 2020.

[16] M. Aguado-Rojas, W. Pasillas-Lépine, and A. Loría, "A switched adaptive observer for extended braking stiffness estimation," in Proc. IEEE American Control Conference, Milwaukee, WI, USA, 2018, pp. 6323-6328.

[17] Y. Liu, "Switching observer design for uncertain nonlinear systems," IEEE Transactions on Automatic Control, vol. 42, no. 12, pp. 16991703, 1997.

[18] A. Alessandri and P. Coletta, "Switching observers for continuous-time and discrete-time linear systems," in 2001 American Control Conference, vol. 3, 2001, pp. 2516-2521.

[19] A. L. Juloski, W. P. M. H. Heemels, and S. Weiland, "Observer design for a class of piecewise linear systems," International Journal of Robust and Nonlinear Control, vol. 17, no. 15, pp. 1387-1404, 2007.

[20] S. Pettersson, "Designing switched observers for switched systems using multiple Lyapunov functions and dwell-time switching," IFAC Proceedings Volumes, vol. 39, no. 5, pp. 18-23, 2006, 2nd IFAC Conference on Analysis and Design of Hybrid Systems.

[21] S. Chaib, D. Boutat, A. Benali, J.-C. Guillot, and J.-P. Barbot, "Observer design for linear switched systems: A common Lyapunov function approach," in IEEE International Conference on Control Applications, 2006, pp. 361-366.

[22] C. Ngo, D. Koenig, O. Sename, and H. Béchart, "A reduced model of three ways catalyst converter and stored oxygen rate estimation using switched observer,' in 2013 European Control Conference, 2013, pp. 3718-3723.

[23] A. Doris, A. L. Juloski, W. P. M. H. Heemels, N. van de Wouw, and H. Nijmeijer, "Switching observer design for an experimental piecewise linear beam system," IFAC Proceedings Volumes, vol. 38, no. 1, pp. 175-180, 2005, 16th IFAC World Congress.

[24] R. Nouailletas, D. Koenig, and E. Mendes, "LMI design of a switched observer with model uncertainty: Application to a hysteresis mechanical system," in 46th IEEE Conference on Decision and Control, 2007, pp. 6298-6303.

[25] A.-Y. Lu and G.-H. Yang, "Secure switched observers for cyber-physical systems under sparse sensor attacks: A set cover approach," IEEE Transactions on Automatic Control, vol. 64, no. 9, pp. 3949-3955, 2019.

[26] Y. Wang and A. F. Lynch, "Multiple time scalings of a multi-output observer form," IEEE Transactions on Automatic Control, vol. 55, no. 4, pp. 966-971, 2010.

[27] M. Sampei and K. Furuta, "On time scaling for nonlinear systems: Application to linearization," IEEE Transactions on Automatic Control, vol. 31 , no. 5, pp. 459-462, 1986.

[28] M. S. Mahmoud and M. G. Singh, Discrete Systems: Analysis, Control and Optimization, ser. Communications and Control Engineering. Springer-Verlag: Berlin Heidelberg, 1984.

[29] Q. Zhang, "Adaptive observer for multiple-input-multiple-output (MIMO) linear time-varying systems," IEEE Transactions on Automatic Control, vol. 47, no. 3, pp. 525-529, 2002.

[30] A. Loría, E. Panteley, and A. Zavala-Río, "Adaptive observers with persistency of excitation for synchronization of chaotic systems," IEEE Transactions on Circuits and Systems I: Regular Papers, vol. 56, no. 12, pp. 2703-2716, 2009.

[31] J. P. Hespanha, "Uniform stability of switched linear systems: extensions of LaSalle's invariance principle," IEEE Transactions on Automatic Control, vol. 49, no. 4, pp. 470-482, 2004.

[32] M. Balde, U. Boscain, and P. Mason, "A note on stability conditions for planar switched systems," International Journal of Control, vol. 82, no. 10 , pp. 1882-1888, 2009.

[33] W. Pasillas-Lépine, "Hybrid modeling and limit cycle analysis for a class of five-phase anti-lock brake algorithms," Vehicle System Dynamics, vol. 44, no. 2, pp. 173-188, 2006.

[34] W. J. Terrell, Stability and stabilization: An Introduction. Princeton, New Jersey: Princeton University Press, 2009.

[35] D. Auroux and J. Blum, "A nudging-based data assimilation method: the back and forth nudging (BFN) algorithm," Nonlinear Processes in Geophysics, vol. 15, no. 2, pp. 305-319, 2008. 